\title{
Testing for Audience Design: a short report
}

\author{
David Pagmar, Caroline Arvidsson \& Julia Uddén
}

\begin{abstract}
In this short report, we present the results from a novel test set-up, aiming to track the practice of Audience Design (AD) in the reference production of Swedish speaking 7 year olds. AD is the conduct of altering one's communicative signal with the receiver of the signal in mind, so that they can easily infer its intended meaning. The results show a distinctive group that does not adapt production in the same manner as in a practice trial prompt for a third party without shared frame of mind. While we controlled for the participant's knowledge of the referential objects of the test, we did not control for the participants assumptions about the world knowledge of the different addresses, which might have altered the result.
\end{abstract}

\section{Introduction}

Following Grice's cooperative principle (1975), a conversationalist ought to have the ambition to form and alter their communicative signal in such a way that a receiver of the signal is able to infer its intended meaning. When a speaker takes into account the perspective and world knowledge of the receiver, as well as any contextual factors affecting them, then the speaker is practicing Audience Design (AD). Consider the following question and reply between interlocutors $\mathrm{A}$ and $\mathrm{B}$ :

\section{A: Which one should I pick? \\ B: Pick Batman}

Whether or not B's answer is an appropriate and/or sufficient response to A's question, depends on the assumptions that $\mathrm{B}$ holds concerning A's knowledge of Batman. If $\mathrm{B}$ has reason to believe that $\mathrm{A}$ knows who Batman is, then $\mathrm{B}$ 's answer is compatible with appropriate $\mathrm{AD}$. If $B$ does not have any reason to believe that $A$ knows who Batman is, then $B$ 's answer is probably not sufficient for pointing out the referent and $\mathrm{B}$ is not practicing appropriate $\mathrm{AD}$.

Appropriate referential communication has been widely discussed and investigated (Clark, et al., 1982; Clark, et al., 1983; Dahlgren \& Sandberg, 2008). In a recent study, Bentdz et al. (submitted) found that young adults take into account the identity of the hearer when forming referential expressions. To our knowledge, no previous studies have investigated this specific ability in children.

A vast number of contextual factors can affect the parameters that need to be altered in a communicative signal, for it to display $\mathrm{AD}$ : conventionality, physical co-presence, world knowledge/frame of reference of the interlocutor, etc. The purpose of this report is to present a novel test set-up that aims to track Audience Design in reference production affected (or not) by the frame of reference of the interlocutor. The aim was to create a task where a child needs 
to assess the frame of reference of an interlocutor to provide sufficient referential expressions. Two measurements were to be obtained from the test: (1) differences on group level between answers to trials where the child does, and does not, share the frame of reference with a interlocutor, and (2) the amount of appropriate answers to trials when the interlocutor does not share the child's frame of reference.

Our own informal pilot data suggested that a child that provides sufficient information to a interlocutor that does not share the child's frame of reference (i.e. describes the visual features of Batman rather than solely provide the proper noun) will also alter their behavior when faced with a interlocutor that shares the child's frame of reference (i.e. simply naming the character when sufficient). The alternative hypothesis is that children generally have not yet developed this pragmatic capacity.

\section{Method}

\section{Participants}

The sample consisted of 49 Swedish speaking children, at the age of 7;0 (observed within two weeks from birth date). The subjects were all participants in the longitudinal study MINT ${ }^{1}$. The sample was slightly skewed towards a high SES demographic, with a large majority of the parents having university diplomas.

\section{The test}

The test was conducted at a laboratory at the Department of Linguistics, Stockholm University. The child was presented with sets of pictures on a computer screen. Each set of pictures included cartoon characters and tv-show hosts, etc., as well as a photograph of a third party that the child was instructed to address (figure 1). The third party was altered between the 7 year old boy and the elderly woman, where the boy did represent a third party that shared the child's frame of reference, while the woman represented one that did not.

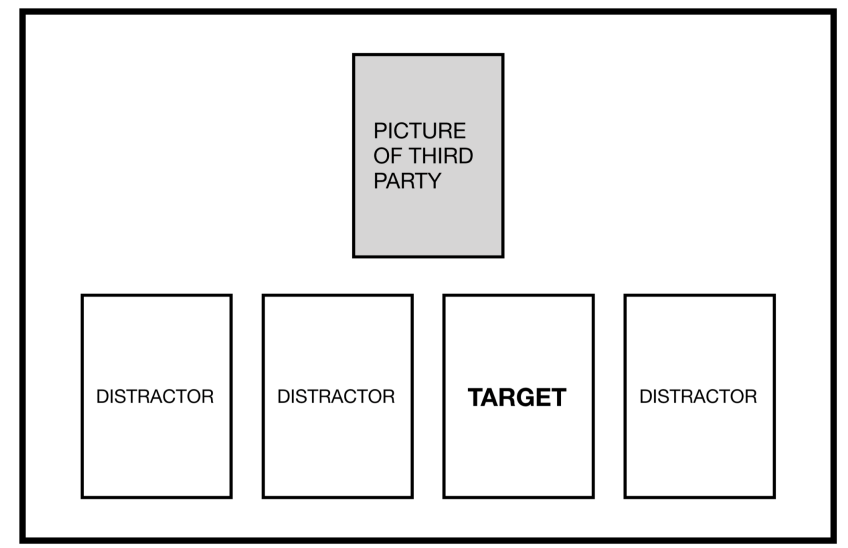

FIGURE 1. The set-up of the pictures that were presented for participants, with a picture of the third party centered at the top, and four pictures (one target in radomized position and three distractors) in a row below the third party.

\footnotetext{
${ }^{1}$ Modelling infant language acquisition from parent-child interaction. The MINT Project was funded by the Marcus and Amalia Wallenberg Foundation (MAW 2011.007) and The Swedish Research Council (2018-01135).
} 
The child was wearing a microphone and an experimenter explained that both the young boy and the elderly woman would stop by the laboratory in a couple of days, and that the child's task was to record messages for them, helping them to pick out certain characters from the potential referents in the pictures. The experimenter said "Can you say something so that (s)he [pointing to the third party] can pick out this image [pointing to one of the potential referents] from these four images?". The characters used in the test were well known to the child (controlled by pre- and post-test surveys, presented below). The child was shown an initial practice trial with pictures of animals, including a white and a brown horse. The experimenter exemplified how to properly refer to one of the horses by saying "the white horse". Each child was exposed to 17 trials in total, with 5 of them including a third party that shared the child's frame of reference (in terms of being the exact same age). The test was delivered semi-manually and the raw data consists of the recorded verbal exchange between the experiment leader and the child.

\section{Pre-and post-test surveys}

An online pre-test survey was sent out to 24 parents of children at the age of 7;0. The parents were asked to mark referents, from a list of 45 popular cartoon characters and tv-show hosts, that they knew that their children were familiar with. A total of 16 parents answered the survey and a list of 24 referents was constructed based on the answers. The list was shown to the parent of the participating child before the test session. The parents were asked to mark the referents that they knew that their child was familiar with. The test leader assembled a participant specific test based on the parents' answers.

Once the child had been exposed to all test trials, a post-test survey was conducted. The referents from the child's specific test were shown once again and the child was asked to name each one. In this way, we controlled for knowledge of the referents, to avoid descriptive answers to test trials due to the child not recognising the referent. All trials where the child did not know the name of a referent were excluded from the analysis.

The final analysis was conducted on 803 test trials ( 833 total trials, 30 excluded due to not passing the post-test survey).

\section{Result and Discussion}

Adept Audience Design for trials with the elderly woman as a third party, relies on describing, rather than naming, the referents in the pictures. Twenty four of the tested children (49\%) consistently named all refrents for trials directed at the elderly woman (figure 2), even though the children were prompted with a description in the initial practise trial. Thus, the result shows that there is a distinctive group that ignores the prompted adept behavior for the third party without shared frame of mind. Furthermore, we found no difference on group level between the two third parties (table 1). 


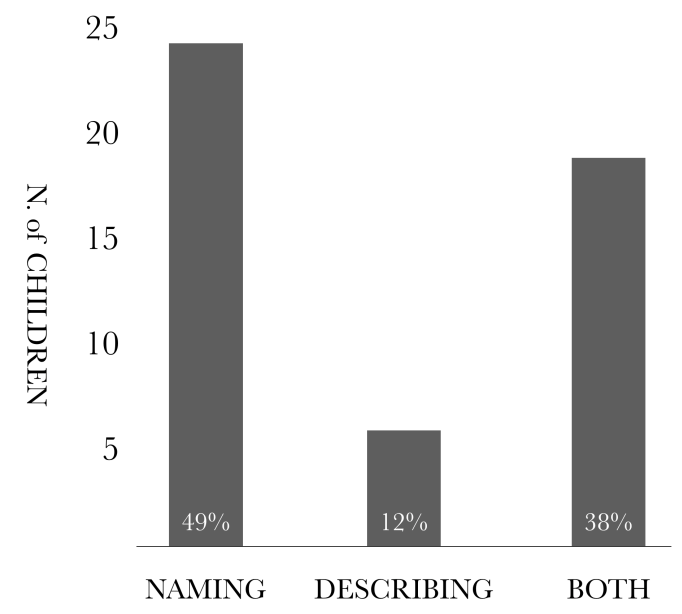

FIGURE 2. Individual behaviours of the participating children, in terms of either only naming, only describing, or some combination of both (either switching between trials or both naming and describing the same trial).

We can see several separate suggestions that could possibly explain the conduct of consistent naming: (1) either costs of abilities related to executive functions, such as working memory and inhibition of one's own perspective, or (2) failed inference regarding interlocutors' knowledge states, resulting in consistently assuming that the elderly woman had knowledge of the referents and hence that describing the targets was redundant, or (3) they did not adapt their utterances in accordance with their assumptions of the interlocutor's frame of reference, which would exemplify an absence of Audience Design. Another possibility is that (4) the children would be able to access sound assumptions about the frame of mind of others, but they will not do it unprompted. A final suggestion would be that (5) the children feel strongly about several of the referents in the pictures and want to name them to show that they know who they are, which could be related to 1 , in terms of inhibition of one's own perspective.

These considerations underline the importance of controlling for participants' assumptions regarding their interlocutors' knowledge in similar paradigms.

\begin{tabular}{|c|c|c|}
\hline THIRD PARTY & NR. OF TRIALS & $\%$ NAMING \\
\hline With shared FoR & $164($ of 240$)$ & $68 \%$ \\
\hline Without shared FoR & 387 (of 563) & $68 \%$ \\
\hline
\end{tabular}

The result of no difference depending on the identity of the third party can be explained in line with the suggestions above, with an additional suggestion for the participants that consistently described the referent, even for the third party that shared the participants frame of reference: a descriptive account of the referent may be a consequence of trying to be accurate in the task of marking referents without taking any social aspects into consideration. It is possible that some or all of these participants could have inferred shared references to 
alter their referential behavior, but that they understood the task as one of marking referents by their attributes.

Nineteen of the tested children (38\%) provided both names and descriptions when marking referents, or switched between the two strategies for different trials directed to the same third party. This result is interesting, although hard to interpret, and future research will have to more thoroughly examine this strategy.

Furthermore, when considering future research, the number of trials should be increased for both trial conditions. The order of trials should also be constructed in such a way that participants are presented with systematically varied initial trials.

In conclusion, the initial run of our novel Audience design test set-up showed a distinct group of 7 year olds, whose strategy of naming referents did not change depending on who they were addressing, even though they were prompted with a descriptive account of a referent. We have suggested five possible explanations, including costs of abilities related to executive function, and failed inference regarding interlocutors' knowledge states. Further research is needed to solidify these findings and to examine possible explanations.

Bentdz, K., Eriksson, S., Schneider, J., Borg, J., Basnakova, J., and Uddén, J. (submitted). Pragmatics as more than core language plus theory of mind: an individual differences study using fMRI. In: Manuscript in preparation.

Clark, H. H. and Murphy, G. L. (1982). Audience Design in Meaning and Reference. In: Advances in Psychology. Ed. by Jean-François Le $\mathrm{Ny}$ and Walter Kintsch. Vol. 9. Language and Comprehension. NorthHolland, pp. 287-299. doi: 10.1016/S0166-4115(09) 60059-5.

Clark, H.H., Schreuder, R., and Buttrick, S. (1983). Common ground at the understanding of demonstrative reference. In Journal of Verbal Learning and Verbal Behavior 22.2, pp. 245-258.

Dahlgren, S., and Sandberg, A. (2008). Referential communication in children with autism spectrum disorder. In Autism: the International Journal of Research and practice 12, pp. 335-48. doi: 10.1177/13623 61308091648 .

Grice, H. P. (1975). Logic and conversation. In Speech acts (pp. 41-58). Brill. 